\title{
On Adiabatic Processes at the Elementary Particle Level
}

\section{Michaud A*}

Senior Researcher, Canada

\begin{abstract}
Analysis of adiabatic processes at the elementary particle level and of the manner in which they correlate with the principle of conservation of energy, the principle of least action and entropy. Analysis of the initial and irreversible adiabatic acceleration sequence of newly created elementary particles and its relation to these principles. Exploration of the consequences if this first initial acceleration sequence is not subject to the principle of conservation.
\end{abstract}

Keywords: Adiabatic process; Acceleration; Kinetic energy; Conservation of energy; Least action; Entropy

\section{Introduction}

Adiabatic processes, the principle of conservation of energy, the principle of least action and entropy have been deeply discussed for centuries and have all been established from experiments carried out at our macroscopic level. However, the degree to which they relate and overlap seems never to have been established to complete satisfaction. They also have been found to generally apply at the submicroscopic level. For example, when applied to the sub microscopic level, adiabatic processes are mostly defined as involving changes of state too rapid for the energy to be released. So, considering that the better part of our knowledge about elementary particles has been acquired in the course of the past century, it seems useful to analyze in depth how these principles fit with this newly acquired knowledge. In physical reality, it is well understood that all naturally occurring processes involving electromagnetic energy at the submicroscopic level naturally tend to increase their energy level until they reach an electromagnetic equilibrium state that they cannot naturally escape, generally related to the concept of entropy. These equilibrium states can be defined as least action equilibrium states because the only way for these states to be reversed to initial unstable states, or to less energetic stable states, is for them to be provided with energy coming from outside these systems.

Fundamentally, all adiabatic processes are also theoretically reversible. But in practice, some of them require such amounts of energy to be reversed that from our human perspective, they can be considered irreversible. Which led to the definition of naturally occurring adiabatic processes as being considered irreversible as viewed from our macroscopic perspective. It is to be noted also that every atom currently in existence in the universe is involved in such reversible adiabatic processes, all of them being ultimately made of a very restricted set of stable, electrically signed and scatterable massive elementary point-like behaving particles, that consequently all are subject to the Coulomb force since they are electrically signed, as we will soon see.

\section{The Principle of Conservation of Energy}

For example, the famous principle of conservation of energy states that the total amount of energy in an isolated system remains constant, meaning that energy can neither be created nor destroyed, but can only transform from one form to another, which precludes the very possibility of energy addition from within an isolated system. Indeed, no experiment ever carried out at our macroscopic level ever gave reason to doubt this principle.

As an example at our macroscopic level, before a $1 \mathrm{~kg}$ mass lying on the ground can be dropped from a height of 1 meter at mean Earth sea level, there is no way but to expend an amount of 9.78 joules of kinetic energy, to elevate it to this height of 1 meter above ground, an amount of energy coming from outside the system made up of this body and the Earth. As the body moves up, the principle of conservation is deemed to be satisfied as this kinetic energy is progressively "converted" to "potential energy", until all of the kinetic energy has disappeared to be replaced by 9.78 joules of "potential energy" when the body finally reaches the height of 1 meter.

As the body is left to accelerate back towards the ground, this "potential energy" is deemed to progressively reconvert to kinetic energy as the body accelerates in free fall towards the ground, an amount of energy that will be released to the environment as the body comes to a sudden stop on the ground. All experimental results confirm that an amount exactly equal to 9.78 joules of energy will be restituted to the environment, exactly compensating the energy initially provided as work to lift this mass to a height of 1 meter above ground.

Let us now examine a similar case at the elementary particle level, that is, the case of a $9.10938188 \mathrm{E}-31 \mathrm{~kg}$ electron stabilized on the mean rest orbital about a proton in a hydrogen atom. It has been extensively confirmed that when this electron is provided with an amount of 13.6 $\mathrm{eV}$ of energy, it will totally escape from the proton, an amount of energy that it will re-accumulate as it eventually accelerates again towards this, or another, ionized hydrogen nucleus (a proton). This re-accumulated amount of exactly $13.6 \mathrm{eV}$ will then be released as an electromagnetic photon as the electron is re-captured in electromagnetic equilibrium on the least action rest orbital of this hydrogen atom, which is deemed to comply with the principle of energy conservation, just as in the case previously examined of the $1 \mathrm{~kg}$ mass. If a less energetic and unstable energy equilibrium state is reached by this electron if provided with less than $13.6 \mathrm{eV}$ of energy, this electron will momentarily meta-stabilize on an authorized orbital further away from the nucleus, but will shortly re-accumulate this exact lesser amount of energy and release it to its surroundings as a less energetic electromagnetic photon as it re-settles back to its more stable higher energy equilibrium state, that is, its maximum entropy least action rest orbital in this atom, which also is deemed to comply with the principle of energy conservation.

*Corresponding author: Michaud A, Senior Researcher, Canada, Tel: 1418624 0608; E-mail: srp2@srpinc.org

Received May 07, 2016; Accepted May 24, 2016; Published June 01, 2016

Citation: Michaud A (2016) On Adiabatic Processes at the Elementary Particle Level. J Phys Math 7: 177. doi:10.4172/2090-0902.1000177

Copyright: (c) 2016 Michaud A. This is an open-access article distributed under the terms of the Creative Commons Attribution License, which permits unrestricted use, distribution, and reproduction in any medium, provided the original author and source are credited. 
In the case of the electron in a hydrogen atom, however, beside this well known amount of $13.6 \mathrm{eV}$ of kinetic energy that can be reversibly provided and recovered, we also know that as long as the electron remains stabilized on the mean rest orbital of the hydrogen atom, it is also permanently adiabatically induced by the Coulomb force with an unreleasable amount of $27.2 \mathrm{eV}$ of kinetic energy, that also reduces to naught without being released to the environment when the electron escapes the hydrogen atom and which also is re-accumulated as the electron is recaptured.

\section{Adiabatic Processes}

We will now put in perspective the various types of adiabatic processes. They fall into three separate categories. The most familiar of which involves increase in energy in a closed system by compression of some gaseous material by mechanical means. The second type concerns naturally occurring increase in energy by solid material compression, and the third involves naturally occurring Coulomb force induced natural levels of adiabatic energy. We will first address the third type, which is less familiar. It can easily be argued that the unreleasable kinetic energy amount of $27.2 \mathrm{eV}$ induced by the Coulomb force at the mean rest orbital of the hydrogen atom will also be consumed as work and be converted to "potential energy" as the electron escapes from the proton, and thus remain conform to the principle of energy conservation, but let us note here that this amount of energy is not induced by compression, but only by the natural action of the Coulomb force between charged particles, at the distance at which both particles naturally fall into some sort of electromagnetic equilibrium, that we will analyze further on. As currently defined, the principle of conservation covers the case of compression induced adiabatic increase in energy generated by mechanical means, because mechanical work is being done to compress the gaseous material used in a closed system, whose momentarily increased energy will be released in the environment when at maximum compression, as applied in various types of refrigeration system or to ignite fuel in diesel engines, for example.

Although theoretically possible, mechanical compression of solid materials to adiabatically increase its Energy level requires such large amounts of mechanical work to be realized that no economically useful purposes can result from such systems. It is however well understood that naturally occurring compression induced increase in energy, unreleasable this time, also naturally occurs in nature in liquid and/or solid materials, as exemplified by the adiabatic heat increase with depth in the Earth mass, which is estimated to reach a stable temperature of about 5100 degrees Kelvin at the center of the Earth ([1], p. 223). In this case, the relation with the principle of conservation becomes more tenuous, because no clearly identifiable amount of work seems to have been involved in this process. Let us note in this regard that all such naturally occurring compression induced adiabatic processes are deemed irreversible and related to entropy.

We are left now with the unresolved case of this $27.2 \mathrm{eV}$ amount of unreleasable energy which is not induced by compression, but by natural application of the Coulomb force. Surprisingly, it seems that at the submicroscopic level, Coulomb force induced unreleasable kinetic energy was never clearly associated to adiabatic processes, nor to the principle of conservation.

\section{The question is: How can this apparent disconnect be reconciled?}

Another question comes to mind at this point: Is this natural Coulomb force acceleration induced adiabatic process also at play in the case of the $1 \mathrm{~kg}$ mass? Calculations that will be carried out further on will confirm that the answer is yes, and the reason why will be clearly identified. We will also see that the increase in unreleasable kinetic energy caused by this naturally occurring adiabatic effect is so infinitesimal with respect to any macroscopic mass used in experiments at approximate sea level, that it is beyond the possibility of any measuring instrument to detect. So it is no surprise that it was never considered for incorporation in the definition of the traditionally macroscopic experiments based principle of conservation of energy.

As it stands, we will see that for a $1 \mathrm{~kg}$ mass accelerating in free fall from a 1 meter height at sea level, this "not-compression-induced" natural adiabatic energy increase is 13 orders of magnitude smaller than the energy contained in 1 gram of mass.

So it seems that with respect to correlating the principle of conservation, the principle of least action and entropy with adiabatic processes, which have been established strictly from experiments carried out at our macroscopic level, we may well be in the same situation currently that Newton was in with respect to relativistic velocities. Just like there was no way he could have even imagined that the measurable mass of bodies could increase with velocities higher than were observable in his time, there was no way that experimentalists could have discovered from macroscopic experiments carried out at approximate sea level that natural adiabatic unreleasable energy increase also occurred during their macroscopic level experiments.

\section{The Principle of Least Action and Entropy}

The principle of least action, on its part, is traditionally related to motion. It has historically been related to identifying the path or trajectory followed by a body or particle that requires the least amount of energy, or the shortest path, to move from one location to another in space. It also applies however to natural equilibrium states that bodies or particles could be captive in, involving motion or not, states into which bodies or particles would remain permanently stabilized, unless provided with energy from some external source to force them out of these least action equilibrium states. It is at this point that the principle of least action can be linked to entropy, and that the principle of conservation can be linked to electromagnetic equilibrium states at the submicroscopic level. But before proceeding to resolve these apparent inconsistencies that become observable at the submicroscopic level, let us summarize what is currently known about this submicroscopic level of physical reality.

\section{Continuity vs. discontinuity with respect to the fundamental interaction laws and the time and space dimensions}

We know for certain that two different interaction laws govern mutual interactions between charged elementary electromagnetic particles. They are the very well-known Coulomb law that governs mutual electric interaction between all charged particles as a function of the inverse square of the distance separating any pair of such particles, and the less familiar magnetic interaction law that governs the mutual magnetic interaction between the same particles as a function of the inverse cube of the distance separating them [2,3]. We also know that the "time dimension" is involved, to account for their motion in the familiar 3 dimensional normal space, because motion of a body or particle minimally involves a change of location in space of the body or particle, which in turn involves that its arrival at a location cannot precede starting to move from its former location, which de facto involves some form of duration of the process, which makes it impossible for some amount of time not to have elapsed between both events. Two fundamental hypotheses have been defined as to how these 
two interaction laws and the time and space dimensions should be defined. The first hypothesis was to consider them as being continuously progressive at the infinitesimal level, metaphorically speaking, just like the continuous microgroove on a vinyl record analogically and faithfully reproduces the complete set of audible frequencies of a music piece, while the second hypothesis was to consider them discontinuous, metaphorically speaking, like the complete set of audible frequencies of a music piece being digitally reproduced by a succession of "dots", so to speak, sufficiently small for the complete sequence to appear continuous as perceived from our macroscopic level when scanned at a sufficient velocity.

The continuously progressive hypothesis is immediately intuitive and was considered the only possibility until Richard Feynman introduced the notion of quantization by means of "virtual photons" in 1949 [4], which allowed using the simpler static Lagrangian calculation method instead of the more elaborate Hamiltonian method to account for interactions between elementary particles. These "virtual photons", that bundle together the Coulomb force and the amount of kinetic energy induced at the particular intensity of the force applicable to each distance considered into individual "virtual exchange quanta", were meant to represent the electric interaction between elementary particles as an exchange of strings of separate mathematical virtual photons.

This method allowed dealing with the electric interaction between charged particles as if the interaction was quantized, being represented by series of instantaneous momentary states, as if frozen in time, as small as required, that would be equivalent at the limit to continuous interaction. The discontinuity idea was soon extended to all interaction laws and even to time and space in some physics circles, which eventually caused the hypothesis that the electric and magnetic forces and even time and space could be discontinuous, that is quantized, at the infinitesimal level, to be adopted by many as completely superseding the continuously progressive hypothesis.

Actually, choosing discontinuity or continuity as characterizing interactions at the infinitesimal level while excluding the other possibility is a philosophical choice that completely determines how the individual will analyze the nature of the fundamental level of physical reality, to the point that the idea of continuously progressive application of force at the infinitesimal level appears unnatural to those choosing discontinuity, and the reverse for those choosing continuity. But there seems to be no reason to reject either method since both hypotheses have qualities and allow possibilities that the other method does not provide; ease of calculation of stable states in the case of discontinuity and ease of motion representation in the case of continuous progression. As surprising as this may seem to some, both views are methods invented by us in trying to understand the nature of the set of particles known to exist at the submicroscopic level and calculate their known interactions. What really matters in reality is acquiring the best possible understanding of what is happening at the submicroscopic level while not coming in contradiction with any experimentally confirmed fact.

Care must be taken, however, not to confuse Feynman's "virtual exchange photons" with real electromagnetic photons, because contrary to "virtual photons", real electromagnetic photons do not bundle together the Coulomb force and the kinetic energy induced by the force, but are made of only kinetic energy in motion at the speed of light [2]. Let us note here that Feynman himself cautioned readers in his seminal 1949 paper [4] about the need not to confuse what he termed "real quanta" and "virtual quanta".

So, while not rejecting the benefits of viewing the fundamental interaction laws and space and time as possibly quantized, we will proceed in this analysis from the continuously progressive approach. Conversion of the described processes can easily be made to the discontinuous quantum electrodynamics method (QED) by those who prefer this option.

\section{Defining the Fundamental Level of Physical Reality}

Let us now put in perspective what this submicroscopic level is made of, energy and matter wise. We know for certain that truly elementary charged particles such as the electron are vanishingly small as perceived from our macroscopic perspective. So small that many even consider them to be point-like with no dimensions in the mathematical sense. But we also know that they are not really dimensionless in this manner, because a transverse cross-section always larger than zero needs to be mathematically involved to correctly account for their scattering encounters, even if we cannot measure a specific volume for them. The reason why a size or volume could never be clearly determined for charged particles behaving point-like, and that have consequently come to be considered elementary, is that the more energetic non-destructive mutual collisions are between 2 electrons, for example, the closer they come to each other's "point-like center" before rebounding, even during head-on collisions, without having reached any unbreachable limit at some distance from their center. A possible cause could be that the material of which electrons are made, which is known to be quantized amounts of electromagnetic energy, that ultimately turns out to be simple kinetic energy as put in perspective explained by Michaud A in [2], may possess some form of elasticity, or some property that we perceive as elasticity, which could account for this behavior. But we still do not know enough about kinetic energy to really identify the cause. The situation is different for protons and neutrons however, that also belong to the same submicroscopic level of physical reality, and for which definite volumes have conclusively been measured, which hinted early on at the possibility that they could have an internal structure. In their case, the rebounding patterns of all incoming particles revealed that they cannot any come closer to their center than a clearly measurable distance of the order of $10 \mathrm{E}-15 \mathrm{~m}$, a limit that remains unbreachable below a very precise energy level of the incoming particles.

After the Stanford Linear Accelerator (SLAC) facility came online in 1966, which was the first high energy accelerator able to accelerate electrons with sufficient energy for them to breach this limit and penetrate inside the previously unbreachable volume of protons and neutrons, it was discovered from the resulting scattering patterns of the incoming electron beams, that two different types of pointlike behaving scatterable charged particles existed inside protons and neutrons. Careful study of the scattering patterns and individual trajectories of the deflected or rebounded electrons, some of which having been directly back scattered in a highly inelastic manner, led to the discovery that both types were charged in opposition and were only marginally more massive than electrons. The positive component was named "up quark", possessing $2 / 3$ of the charge of the positron, and the negative component was named "down quark", possessing $1 / 3$ of the charge of the electron. No other scatterable inner components were ever discovered inside nucleons. This is how it was discovered that the scatterable inner structure of the proton is made of 2 up quarks and 1 down quark (uud), whose sum of fractional charges resolves to the known unit positive charge of the proton, while the scatterable inner structure of the neutron is made of 1 up quark and 2 down quarks (udd), whose sum of fractional charges resolves to the known null charge of the neutron (Table 1). A series of papers were issued at the SLAC facility to account for this discovery, explained in [5] being one of them.

Given that all atoms in existence are made exclusively of electrons, 


\begin{tabular}{|c|c|c|c|c|}
\hline Particle & Estimated mass & Mass in Kg & Charge & Ref. \\
\hline Electron & $0.511 \mathrm{MeV} / \mathrm{c}^{2}$ & $9.10938188 \mathrm{E}-31$ & -1 & {$[24]$} \\
\hline Up quark & $\begin{array}{c}1.5 t o 5 \mathrm{MeV} / \mathrm{c}^{2} \\
\text { (Experimental Estimation) }\end{array}$ & $2.04961092 \mathrm{E}-30$ & $+2 / 3$ & {$[20]$} \\
\hline Down quark & $\begin{array}{c}\text { 3to } 9 \mathrm{MeV} / \mathrm{c}^{2} \\
(\text { Experimental Estimation) }\end{array}$ & $8.19844378 \mathrm{E}-30$ & $-1 / 3$ & {$[20]$} \\
\hline Proton & $938.272013 \mathrm{MeV} / \mathrm{c}^{2}$ & $1.67262158 \mathrm{E}-27$ & +1 & {$[24]$} \\
\hline Neutron & $939.5653346 \mathrm{MeV} / \mathrm{c}^{2}$ & $1.67492716 \mathrm{E}-27$ & 0 & {$[24]$} \\
\hline
\end{tabular}

Table 1: Masses and charges of elementary components in atoms.

protons and neutrons, the latter containing only scatterable charged and massive up and down quarks, which behave point-like in all circumstances just like electrons, we consequently know that all existing point-like behaving building blocks of matter are subject to the Coulomb force since they all have electric charges. Up and down quarks are the building blocks of protons and neutrons, which are the building blocks of all atomic nuclei, about which, when in least action equilibrium states, a number of electrons equal to the number of protons in the nuclei, settle in a series of least action equilibrium layers, which determines the physical volume of atoms.

Besides the electron, the up quark and the down quark, two other stable point-like behaving particles belong to the same stable set. They are the positron and the electromagnetic photon. The doubleparticle electromagnetic photon as hypothesized by Louis de Broglie is described in [2]. The positron will be described further on.

\section{The Least Action Equilibrium States of all Previously Existing Particles}

Attention is not generally drawn to the fact that prior to any experiment being carried out at our macroscopic level to confirm the principle of conservation of energy, the system considered is always previously stabilized into the least action equilibrium state of some reversible process. Actually, this situation applies to any transformation involving matter and energy in all systems that are within our reach to experiment with at the macroscopic level. We observe that all bodies at the surface of the Earth are usually in various states of least action equilibrium of various reversible processes with respect to the center of the Earth. All components of our atmosphere are globally in various states of least action equilibrium of reversible processes with respect to the center of the Earth. We also observe that our planet and all other planets in the Solar system are in states of least action equilibrium of reversible processes with respect to the Sun, theoretical in the latter case due to the incredibly huge amounts of energy that would be required to reverse the process, and so on..., meaning that energy must be provided from outside these isolated systems for these bodies to stray from these least action equilibrium states of motion, otherwise, they will permanently remain in these states with respect to the local intensity of the gravitational gradient.

Regarding least action equilibrium states in atoms, the reason was never clearly identified as to why negatively charged electrons stabilize on a series of least action equilibrium layers at specific distances from nuclei instead of crashing on them, contrary to prima facie expectation, given that they are attracted as a function of the inverse square of the distance separating them from the positively charged nuclei by the Coulomb force.

Why is it that a negative electron and a positive positron, that mutually capture in a metastable positronium configuration, succeed in spiraling inwards until they actually meet, causing them to convert to various photon states, while a negative electron and a positive proton always settle in a stable configuration at a very precise mean distance to form a stable hydrogen atom, a mean distance known to be exactly equal to $5.291772083 \mathrm{E}-11 \mathrm{~m}$ ?

We will see that both the electric and the magnetic interaction laws previously mentioned have to be involved in the process. The inverse square law that causes the electric aspect of charged elementary particles to interact, that is, the Coulomb law, is very familiar as already mentioned, but the inverse cube law that causes the magnetic aspects of the same point-like behaving elementary particles to interact is much less familiar. A major telltale that these two orders of force are simultaneously and permanently involved between point-like behaving electrons is the covalent electronic link that unites atoms into molecules, involving mutual capture of 2 electrons, one from the outer electronic layer of each atom involved in such covalent bounding, and the fact that electronic layers can be filled only by electron pairs interacting in relative antiparallel spin orientation.

Indeed, the only possible way for two electrons to so paradoxically attract when they are very close to each other despite their mutual electrostatic repulsion (obeying the inverse square law), can only be that another force, acting in attraction, would simultaneously be locally at play between them, that would obey a higher order exponential law than the inverse square law, and that consequently would overcome the electric inverse square repulsion when the particles are close enough to each other. Figure 1 illustrates how these simultaneously acting forces relate:

A direct confirmation of this inverse cube interaction law between the magnetic aspects of electrons was very recently experimentally obtained by Shlomi Kotler and his team between the magnetic aspects of two electrons, as reported in an article accepted for publication in Nature magazine in April 2014, titled "Measurement of the magnetic interaction between two bound electrons of two separate ions" [6]. This confirmed inverse cube interaction law is precisely such a higher order exponential law. Electrons and the up and down quarks making up the internal structure of protons, possessing both an electric aspect (obeying the inverse square interaction law) and a magnetic aspect (obeying the inverse cube interaction law), since they are charged, thus electromagnetic in nature, it can forcefully be asserted that the states of equilibrium of electronic layers in atoms mandatorily have to

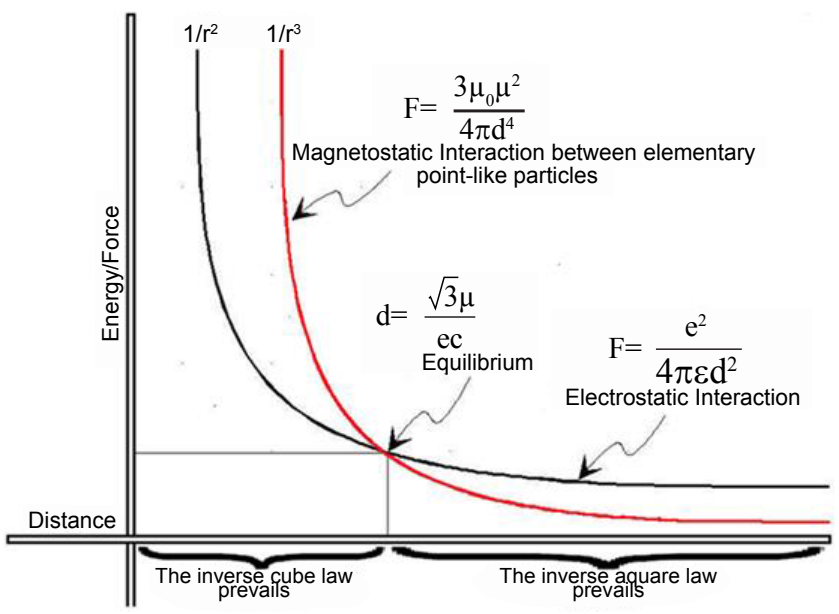

Figure 1: Intersecting inverse square and inverse cube force interaction laws 
involve both types of interaction. It can also forcefully be asserted that both poles of their magnetic field geometrically coincide by structure, given that they behave point-like in all circumstances. A confirming experiment carried out with magnets similarly magnetized also allows concluding that both poles cannot be present simultaneously, but only in alternance [3]. Furthermore, the dynamic inner LC structure of the energy contained in electrons, that can be established in the 3-spaces space geometry, reveals that this alternance is linked to the frequency of the amount of energy making up the rest mass of the electron [2,7].

This LC oscillation clearly establishes that the magnetic aspect of localized elementary electromagnetic particles is made up of two distinct time-wise phases in the 3-spaces geometry. The first phase consists in the particle's magnetic field cyclically spherically expanding from zero presence to maximum presence, followed by a second phase as it spherically regresses to zero presence. Given their point-like behavior in all interaction circumstances, the magnetic interaction between two electrons, or between an electron and a positron, for example, is thus bound to obey by structure a far fields interaction law since it involves two interacting energy spheres, which means that their magnetic relation will obey the following far fields equation, that was established from the data collected during the previously mention experiment [3]:

$$
\mathrm{F}=\frac{3 \mu_{0} \mu^{2}}{4 \pi \mathrm{d}^{4}}
$$

Consequently, in the 3-spaces geometry, relative parallel spin alignment between two electrons will occur when the magnetic presence of the energy of both particles is synchronously expanding and regressing at the same time, which amounts to an inverse cube magnetic repulsion with distance between the two magnetic spheres.

Conversely, relative anti-parallel spin alignment will occur when the magnetic energy presence of one electron is synchronously in its expansion phase while that of the other electron is in its regression phase, which amounts to an inverse cube magnetic attraction with distance between both particles.

Considering that the rest energy of both electron and positron oscillates at the exact same frequency, it is consequently quite understandable that when in positronium anti-parallel spin configuration, both particles will easily synchronize to eventually be overwhelmed by the inverse cube magnetic attraction, that then comes in addition to the inverse square electric attraction between both particles when the electric vs. magnetic equilibrium point is reached (Figure 1), to then spiral inwards and finally meet and convert to photon states.

Even when in parallel spin positronium configuration, when the electric vs. magnetic equilibrium point is reached, it is also quite understandable that at this equilibrium distance, they would tend to easily switch to anti-parallel spin alignment, like two magnets always do even at our macroscopic level in similar circumstances, to then spiral inwards until they meet, the key factor allowing both particles to attract until they collide being the fact that the energies of both particles oscillate at the exact same frequency, which will maintain antiparallel synchronization during the whole process. When dealing with an isolated hydrogen atom, however, the situation is quite different, given the huge differences in oscillating frequencies between the magnetic cycle of the captive electron and those of the much more energetic central proton components.

At this point, time has come to introduce the description of the carrying energy of electromagnetic particles. All point-like behaving scatterable elementary particles such as the electron, the positron, the up quark and the down quark are known to possess a fixed rest mass, whose LC representations in the 3-spaces model are detailed in [7] for the electron and positron, and in [8] for the up and down quarks.

The velocity in space of these particles depends on the amount of kinetic energy that they possess in excess of the energy captive of their rest mass. In the 3-spaces space geometry, it can be shown that this carrying energy remains separate from the rest mass energy of the particle, and also possesses the same LC structure as that of free moving photons, hence the name "carrier-photon" that can be given to them, on account of the fact that it can be shown mathematically that if they were not carrying the inert rest mass of the particle, they would move at the speed of light, just like free moving photons [2,9].

The same analysis leading to the establishment of the LC equations for carrier-photons [2,9], that correlates the conclusions of Paul Marmet that the magnetic field of a moving electron increases as the square of its velocity [10], with Walter Kaufmann's experimental results regarding the difference between transverse and longitudinal inertia of electrons accelerated to relativistic velocities [11], reveals that half of a carrier-photon's energy systematically converts to electromagnetic mass, possessing omnidirectional inertia just like the rest mass of the carried particle.

At low non-relativistic velocities, the transverse and longitudinal inertia of electrons do not reveal any measurable difference due to the infinitesimal contribution of such low amounts of added kinetic energy. However, Kaufmann's experiments with much higher added kinetic energy amounts, reveal that the difference has to be linked to the electron carrying energy, since the rest mass of the electron is invariant.

In 2003, Paul Marmet successfully linked the velocity related relativistic mass increase of electrons to a simultaneous increase of their magnetic field [10]. Specific calculations then showed that the difference between the electron rest mass and its relativistic mass at any velocity corresponds to an increase in mass equal to half of the induced kinetic energy divided by the square of the speed of light, which is exactly equal to the velocity related "magnetic mass increment" that can be calculated from Marmet's discovery as analyzed in references $[2,9]$.

Since this added "magnetic mass" displays omnidirectional inertia just like the electron rest mass to which it must be added, it can also be measured by transverse interaction. This leaves as the only possible candidate to explain the difference between longitudinal and transverse inertia, the translational half of the kinetic energy provided to the electron, whose longitudinal inertia can be measured as proven by Einstein's photoelectric proof, but whose transverse inertia apparently cannot as shown by Kaufmann's experiments.

This leads to the unavoidable conclusion that the unidirectional half of the energy of a carrier-photon, which propels the total velocity related instantaneous mass of the electron, can only be impervious to transverse interaction, and that the instantaneous relativistic mass of a moving particle can be measured directly only by means of transverse interaction since longitudinal inertia does not allow distinguishing the rest mass of the particle from the velocity related mass contribution of half of its carrying energy.

Since that in the 3-spaces model space geometry, omnidirectional inertia is an exclusive property of the LC oscillating half of the carrierphoton's energy [2], this means that carrier-photons also possess a magnetic aspect that oscillates between zero and maximum magnetic 
presence at the frequency linked to the total amount of energy of the carrier-photon.

It can be hypothesized at this point that to explain the natural mean rest orbital stability in a hydrogen atom, when the electron comes closer to the proton than this mean rest orbital, the magnetic interaction between the proton components and the electron could, for reasons that we will identify, always become "preponderantly repulsive" to the point of always overcoming the electric attraction between electron and proton, during such moments of closer proximity and repel the electron, whereas if the electron got farther away than the mean rest orbital, the electrostatic attraction would dominate again as illustrated with Figure 1, bringing it back, so that the motion of the electron would generally stabilize about the mean equilibrium distance at which the Quantum Mechanics statistical spread averages out.

It goes without saying that such an electromagnetic equilibrium distance could exist only if the averaged out magnetic interaction between nucleus and electron always became preponderantly repulsive (never preponderantly attractive) whenever the electron comes closer to the nucleus than its known least action mean rest orbital.

In this regard, the spherical expansion-regression dynamic structure of elementary particles' magnetic behavior which becomes so obvious in the 3-spaces model, does offer a wonderful surprise! We will now demonstrate that in this model, the averaged out magnetic interaction between nucleons and electronic escorts can only become preponderantly repulsive whenever an electron comes closer to a nucleus than its least action mean rest orbital, which can only force electrons to stabilize at specific equilibrium mean distances from atomic nuclei.

Let us first make an inventory of the various electromagnetic components involved in an isolated hydrogen atom. For the electron, we are dealing with two distinct electromagnetic quantities, the electron proper with its $0.5109989 \mathrm{MeV}$ rest mass energy (Table 1) and its unreleasable $27.2 \mathrm{eV}$ carrier-photon (Table 2), whose value locally adiabatically varies with the distance between electron and proton and which is permanently induced at exactly this value when the electron happens to be located precisely at the mean rest orbital distance from the proton. Given that a first approximation will be sufficient to explain the equilibrium mechanics, we will proceed by taking into account only the magnetic field of the electron, since that of its carrier-photon is relatively negligible.

As for the proton, the situation is much more complex, and somewhat unexpected. Even if the relative angular parallax of the proton diameter can make it appear point-like as perceived from the electron perspective, given the distance separating the rest orbital from the proton, which means that far fields magnetic interaction equation (1) can be considered to generally apply, each cycle of the frequencies of the 6 individual proton components will still interact with those of the electron and its carrier-photon.

While the energies contained in the rest masses of the up and down quarks are respectively $1.1497475 \mathrm{MeV}$ for each of the up quarks and

\begin{tabular}{|l|c|c|}
\hline Particle & Energy & References \\
\hline Electron carrier-Photon at the mean ground stable orbital & $\begin{array}{c}27.2 \mathrm{eV} \\
\text { (Exact) }\end{array}$ & Equation (10) \\
\hline Up or down quark carrier-Photon inside the Proton & $\begin{array}{c}310.457837 \mathrm{MeV} \\
\text { (Approximate) }\end{array}$ & [8] \\
\hline
\end{tabular}

Table 2: Energy of the carrier-photons of the point-like behaving massive components of the hydrogen atom.
4.5989902 MeV for the single down quark (Table 1), as determined in reference [8], the unreleasable energy of their 3 carrier-photons each are equal to $310.457837 \mathrm{MeV}$ as determined in the same reference (Table 2), which represents about 300 times more energy than that of the rest masses of the particles that they carry. This means that it is the magnetic fields of the 3 quarks carrier-photons that will interacts the most strongly with the electron, while the rest mass energy of the quarks themselves is negligible with regard to interaction with the orbiting electron!

The minor contribution of the up and down valence quarks to the proton spin has in fact been demonstrated in 1995 at the SLAC facility, which is coherent with this conclusion of the present model.

\section{Relativistic Velocities Mass Increase Dependence versus Stabilized Unreleasable Adiabatic Energy Mass Increase Dependence}

One particular issue now needs to be clarified before proceeding further, regarding the velocity dependence of "relativistic mass increase". When an electron is moving freely, its momentary "relativistic" mass increment can truly be said to be velocity dependent, but it must be emphasized here that this velocity is itself dependent on the amount of carrying-energy that the electron possesses in excess of the energy making up its invariant rest mass [2,9].

It must also be made clear that when an electron is captive on an atom's orbital, even though the local electromagnetic equilibrium may not allow it to move, this mass increment due to the permanent presence of the adiabatic unreleasable amount of carrying-energy electrostatically induced in it by the Coulomb force as a function of the inverse square of the distance separating it from the nucleus, is still present, even if it cannot now be related to any relativistic velocity.

So, experimental reality reveals that in such cases, just as in the case of freely moving electrons, this mass increment is directly "carryingenergy level dependent", and "not directly velocity dependent", even if the latter phrase is not misleading when referring to an electron in free motion, because velocity is itself dependent on whether or not the local electromagnetic equilibrium will allow it to be expressed, even if the particle carrying-energy would be sufficient to sustain said velocity. So every time velocity dependence is being mentioned in reference to relativistic mass or relativistic mass increment, the more fundamental "carrying-energy dependence" aspect of the relation must be kept in mind.

\section{Correlating the Frequencies of the Hydrogen Atom Components}

So to keep the demonstration simple, this allows us to assume that the electron captive on the rest orbital in "an isolated hydrogen atom" is free to move at the relativistic velocity allowed by its carrier-photon.

Let us now determine the number of times that the electron rest mass energy will magnetically oscillate from zero to maximum and back to zero during one complete orbit about the proton in this isolated hydrogen atom. The precise distance between the nucleus and this mean rest orbital is very well known and equal to $\mathrm{a}_{\mathrm{o}}=5.291772083 \mathrm{E}-11$ $\mathrm{m}$, giving a complete orbital length of $2 \pi \mathrm{a}_{\mathrm{o}}=3.32491846 \mathrm{E}-10 \mathrm{~m}$.

The relativistic velocity of the electron on such an orbit being $2187647.56821 \mathrm{~m} / \mathrm{s}$, the time required for one orbit to be completed will be $1.51986 \mathrm{E}-16 \mathrm{~s}$. Given that the frequency of the electron rest mass energy is $1.235589976 \mathrm{E} 20 \mathrm{~Hz}$, this energy will cycle 18779.23781 times while one orbit is being completed. 
On the other hand, in the 3-spaces model, the energy of the unreleasable energy of each carrier-photon of the up and down quarks in the proton is $310.457837 \mathrm{MeV}$, [8], which resolves to a value in joules of $4.974082389 \mathrm{E}-11 \mathrm{j}$, corresponding to a frequency of 7.506837869E22 $\mathrm{Hz}$. So for each orbit of the electron, the energy of each quark carrierphoton will cycle 11409342.2 times. This means that during each complete magnetic cycle of the electron rest mass energy, the energy of each quark carrier-photon will cycle 607.5508878 times.

Let us now examine Figure 2 illustrating an arbitrary segment corresponding to 6 of the 18779.23781 cycles that the magnetic energy of the electron will complete during one orbit, with one segment further isolated, representing one occurrence of the electron magnetic presence:

The upper sequence in Figure 2 represents the axial travel of the electron about its average distance from the nucleus, corresponding to the corrected and limited statistical spread that the wave function should be restricted to, to correctly represent physical reality (Figure 3 ). The central sequence represents the variation in intensity of the "magnetic presence" of the electron rest mass energy during each of its cycles. The lower sequence represents the 607.5508878 intensity variations of the "magnetic presence" of the nucleus carrier-photons energy, that occur during each magnetic cycle of the electron.

Obviously, the intensities (and number of cycles per second for the proton) are not represented to scale, since the energy of each quark carrier-photon is about 600 times that of the electron, and that 2 of the

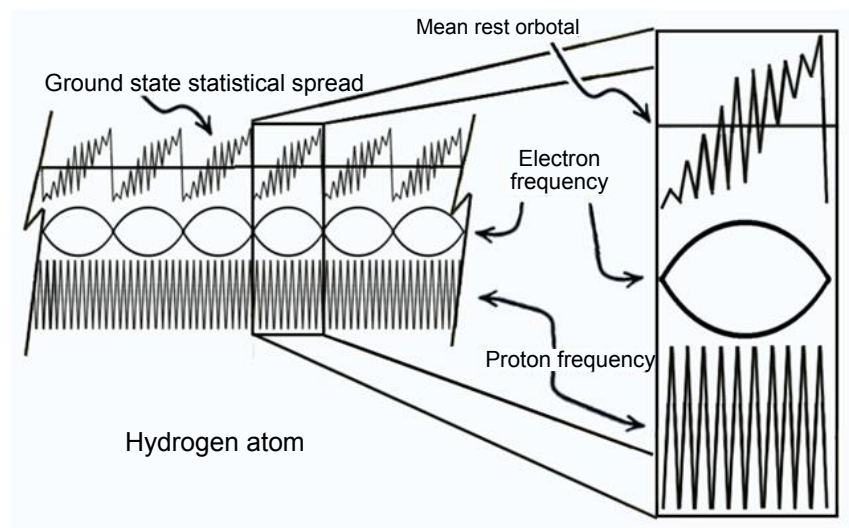

Figure 2: Representation of the conflicting magnetic fields frequencies in the hydrogen atom.

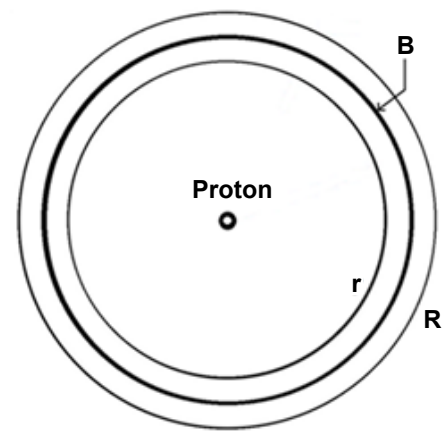

Figure 3: Maximum extension of the physically possible electron location statistical spread on the rest orbital of the isolated hydrogen atom. carrier-photons of the proton are always in parallel spin alignment by structure with respect to the third.

For simplicity's sake, we will ignore here the magnetic drift of the quarks carrier-photons' unreleasable energy inherent to the fact that they are moving on such narrow closed orbits, a drift that can only increase yet more the "magnetic presence" of the particles involved during each cycle, as analyzed in reference [12].

Examining the isolated segment in Figure 2, one can easily visualize that at the beginning of the expansion phase of magnetic presence the oscillating energy of the electron, which has very little inertia with respect to the nucleus, the electron will be repelled according to the inverse cube magnetic interaction law a certain distance due to the intensity of the magnetic nucleus energy presence increasing towards its maximum during the first part of the first of the latter's 607 cycles, which comes in opposition (that is, in parallel spin alignment) to the presence of the electron magnetic energy which also is in its much more slowly increasing phase.

One can also easily understand that when the magnetic presence of this first cycle of the nucleus energy will start diminishing towards zero after having peaked to its maximum, then becoming attractive, that is, in anti-parallel spin alignment with respect to the still increasing electron magnetic presence, that there will be magnetic attraction between the electron and the nucleus during this nucleus magnetic presence diminishing phase as a function of the inverse cube magnetic interaction law, in addition to the electrostatic attraction.

It is here that the enigma unravels, because, given that the magnetic force obeys the same inverse cube interaction law with distance in attraction and repulsion and that the electron is now located farther away than at the beginning of the magnetic increasing phase of the proton, then this force will be acting more weakly as a function of the inverse cube relation on the electron at the precise moment when the relative spin switches to anti-parallel attraction than when it started the repelling phase, while the electron was closer to the nucleus.

Consequently, there will be a "physical impossibility" for the electron to be brought all the way back to the distance it was at, at the beginning of the previous increasing magnetic presence of the nucleus energy, because the duration of the attractive phase is the same by structure as that of the repulsive phase.

The same situation will be reproduced for each of the remaining 606 cycles of the nucleus carrier-photons magnetic presence. The result can only be a progressive motion of the electron away from the nucleus, made up of very precise axial to and fro motions until the intensity of the magnetic presence of the electron energy becomes too small and finally momentarily falls to zero, moment during which all magnetic interaction having disappeared, the electron will fall freely towards the proton, now obeying the only force still active, which is the permanently active inverse square electrostatic force, until the intensity of the magnetic presence of the energy of the electron becomes sufficient again at the beginning of the following increasing phase of its magnetic cycle, for the predominantly repulsive magnetic interaction to start dominating again when the electron come closer than the mean rest orbital.

So this process of cyclic variation of the electromagnetic equilibrium distance between nucleus and electron can only force the electron to continuously move axially in order to progressively occupy all of the physically possible locations of the statistical distribution covered by the Quantum Mechanics wave equation, but with the restriction that 
this spread is mandatorily restricted to the only set of locations allowed by the inertia of the electron as it sustains transverse accelerations and decelerations, while being maintained in a stable manner at an average distance from the nucleus corresponding to the mean hydrogen rest orbital by the opposing and permanent electrostatic attraction and the preponderantly repulsive magnetic interaction just analyzed.

In summary, the probabilistic spread of possible locations of the electron in motion in an isolated hydrogen atom is traditionally represented by this form of the wave equation:

$$
\int_{-\infty}^{\infty}|\varnothing|^{2} \mathrm{dxdyd} z=1
$$

Which is a form representing the statistical spread as theoretically reaching infinity. But to really account for the limits imposed by the inertia of the electron during transverse accelerations and decelerations, equation (2) should then be modified to the following form to more closely reflect physical reality:

$$
\int_{-d}^{+d}|\varnothing| d x d y d z=1
$$

with reference to Figure 3, where "B" represents the mean rest orbital, in equation (3) "d" represents the farthest transverse distance about the mean equilibrium rest orbital that this limiting factor imposes on the localized electron in motion, "- $d$ " referring to radius " $r$ " and " $+\mathrm{d}$ " referring to radius " $\mathrm{R}$ ". When no outside forces are applied, this statistical spread can be expected to tend being limited to a circular axial two-dimensional band circling the nucleus, with the set of most probable locations averaging out at the mean rest orbital radius.

Of course, due to interactions with surrounding matter, in physical reality, this band is more likely to spread at the limit to a $3 \mathrm{D}$ volume circumscribed by the surfaces of two concentric spheres whose inner and outer radii will respectively be $r$ and $R$. So it is inside this volume exclusively that the normalization condition must apply, any other location in space becoming physically impossible, unless more energy is provided to the electron.

Let us note also that even when electrons are immobilized on their orbitals by local electromagnetic equilibrium states involving other electrons or atoms, the same electric vs. magnetic repulsive interaction is bound to apply all the same.

The electron orbital ring structure predicted by the 3 -spaces model (Figure 3 ) is clearly recognizable in projections recorded during an experiment carried out by Stodolna et al. in 2013, that show clearly defined separate rings corresponding to the electron rest orbital and the further away metastable orbitals to which the electron repeatedly jumped during the experiment with a hydrogen atom. This article, titled "Hydrogen Atoms under Magnification: Direct Observation of the Nodal Structure of Stark States" is noted [13].

Now that the electromagnetic equilibrium mechanics of the hydrogen atom has been clarified, this brings us to consider the first time a newly created electron accelerates for the first time of its existence to reach such an equilibrium state of maximum entropy in a given system.

\section{The Creation of Point-like Behaving Elementary Particles}

Now that the principle of conservation has been correlated with natural adiabatic processes, that the principle of least action and entropy have been linked to normal least action electromagnetic equilibrium states at the atomic level, and finally that the stability of electron equilibrium states in atoms have been summarily analyzed, the next issue to be addressed is the origin of these point-like behaving elementary particles that are known to be stable, charged and massive, which are the positron, the electron, the up quark and the down quark, the last 3 being known to be the only scatterable point-like behaving building blocks of all existing atoms. We will first address the case of electrons and positrons. In a previous paper, the manner in which electromagnetic photons come into being has been exhaustively analyzed [2]. It is also well verified that electrons and positrons can be created from the destabilization of electromagnetic photons possessing a minimum energy level of $1.022 \mathrm{MeV}$ [7]. In 1933, Blackett and Occhialini proved experimentally that cosmic radiation byproduct massless electromagnetic photons of energy $1.022 \mathrm{MeV}$ or more will spontaneously convert to massive electron/positron pairs when grazing atomic nuclei [14], a process that was named "materialization". Moreover, a team led by Kirk McDonald confirmed with experiment \# 144 , carried out in 1997 at the Stanford Linear Accelerator, that by converging two sufficiently concentrated photons beams toward a single point in space, one beam being made up of massless electromagnetic photons exceeding the $1.022 \mathrm{MeV}$ threshold, massive electron/positron pairs were created without any atomic nuclei being close by [15].

It was also exhaustively demonstrated that positrons and electrons are totally identical, except for the sign of their charges, both particles having the exact same invariant rest mass of $9.10938188 \mathrm{E}-31 \mathrm{~kg}$, that is, $0.511 \mathrm{MeV} / \mathrm{c}^{2}$, which is exactly half the energy of the lowest energy photon that can convert to a pair of these particles. When a photon being converted possesses more than this $1.022 \mathrm{MeV}$ energy threshold, the energy in excess directly determines the relative velocities in opposite directions of both particles in space after materialization [16].

Of course, it goes without saying that most if not all of these pair creation events do not occur at least action rest orbital distance from a handily available ionized hydrogen nucleus (a proton), particularly in cases similar to the process confirmed by the Kirk McDonald et al. From the universe at large, the main source of photons of energy $1.022 \mathrm{MeV}$ or more that we can detect involves so-called cosmic "radiation", which is in fact mostly made up of extremely energetic protons moving at close to light velocity. When such hyper-energetic protons collide with atomic nuclei in planets' atmospheres or ground surfaces, as observed by Blackett and Occhialini, part of their kinetic energy is released as such highly energetic free-moving photons due to the sudden slowing down that they suffer during such encounters, termed bremmsstrahlung radiation. Other known sources are gamma radiation from radioactive decay and stars' and corona's activity $[17,18]$

So this means that electrons created during such pair creation processes, if they survive the ordeal of their creation without recombining with their companion positron to reconvert to free moving massless electromagnetic photons, will eventually be attracted for the first time of their individual existence to protons to form hydrogen atoms, will settle on their least action equilibrium ground state orbital with their adiabatically stabilized and unreleasable $27.2 \mathrm{eV}$ Coulomb induced energy, and will each release a $13.6 \mathrm{eV}$ electromagnetic photon, which is the translational energy that it accumulated while accelerating towards the proton, and this, without having been previously provided with this energy.

It may be argued here that to even get away from the positron of the pair in the process of being created, the electron must possess some translational energy already, made up of the energy that the mother photon possessed in excess of the $1.022 \mathrm{MeV}$ minimum that converted to the masses of the two newly created particles. And why not $13.6 \mathrm{eV}$, 
and a little more in fact, that would leave no doubt that both particles will really escape from each other. It remains that no amount of this residual translational energy coming from the mother photon was ever expended to pull this new electron away from any proton. We also know that no work is done during such pair production processes, because the sum of the energies making up the newly created electron and positron plus the residual translational energy that causes them to move away from each other has been experimentally proven to be exactly equal to the energy contained in the mother photon before conversion [7].

This means that a newly created electron will eventually accelerate for the first time of its own existence, will stabilize with a new and unreleasable adiabatically induced energy amount of $27.2 \mathrm{eV}$ as it reaches the least action rest orbital of a hydrogen atom, and will finally release an amount of $13.6 \mathrm{eV}$ of new energy, for a grand total of 40.8 $\mathrm{eV}$ that never was compensated by a previously expended equivalent amount of work.

This is an example of an "initial irreversible adiabatic acceleration sequence" of a newly created electron, because forever after, $13.6 \mathrm{eV}$ of energy will reversibly have to be provided for this electron for it to move away and escape from the proton before it will naturally re-accelerate and re-accumulate this amount of energy, which will be released as it is recaptured on the rest orbital of a proton, while restabilizing with the $27.2 \mathrm{eV}$ Coulomb induced unreleasable adiabatic energy quantum.

All probabilities would then be that the initial and irreversible adiabatic acceleration sequence of such a newly created electron towards its first least action rest state would by definition not be subject to the principle of conservation of energy, which it consequently could not possibly violate, because it answers all criteria of an irreversible adiabatic process, considering that all processes proven to obey the principle of conservation are reversible. Moreover, if later, in the course of the usual subsequent processes of repeated liberations and recaptures, this electron ever happens to stabilize on an electronic layer requiring more intense energy than it ever reached even once before, then by very definition, the added amount of energy concerned, required to reach for the first time this more energetic least action electromagnetic equilibrium state, which would be induced by this further irreversible adiabatic additional first time acceleration sequence, would also not be subject to the principle of conservation without violating it.

This will obviously be true up to and including the most energetic inner orbital that an electron can naturally reach about the densest atom in existence.

What is acceleration, by the way?

Before analyzing this case further and addressing the case of the up and down quarks, let us now clarify the actual mechanics of natural freefall acceleration, during which a charged particle's kinetic energy increases, a process that would of course be the same for the "initial irreversible adiabatic acceleration sequence" of a newly created electron and for the natural acceleration of an electron that was previously chased away from a proton, the latter case answering all criteria of a "reversible adiabatic process" with regard to the unreleasable $27.2 \mathrm{eV}$, and all criteria of the principle of conservation, with regards to its 13.6 $\mathrm{eV}$ of releasable energy.

\section{Defining acceleration}

What better way to clarify such an issue but to give a practical example, which also applies to all possible cases of free-fall acceleration, "free-fall" meaning here 'falling' in the sense of "being subjected to a force inducing energy as a function of the inverse square of the diminishing distance separating mutually attracting bodies", such as the Coulomb force or the gravitational force.

Let us dwell for a moment on the concept of force, an oftentimes assumed axiom about which is that it "cannot move faster than $\mathrm{c}^{\prime}$ or that it "can move only at c".

Let us consider that the Coulomb force, which is verifiably in action between any pair of charged particles, and whose nature and origin still are a mystery, is known to be in continuous infinitesimally progressive decreasing or increasing action, depending on whether the charges repel or attract, as a function of the inverse square of the distance between the particles involved, irrespective of the distance between them, which means that the force does not "travel" or "move" as such between them, but is "permanently present" by structure between charged particles.

We know that the Coulomb force is however the cause of the acceleration of charged particles towards or away from each other; or more precisely formulated, it is the cause of the induction of kinetic energy in charged particles, at least a part of which will be unidirectional directed towards the other particle in case of attraction, and in the opposite direction in case of repulsion, which is the translational energy that naturally manifests its presence by propelling particles at measurable velocities when external electromagnetic constraints do not partially or even completely prevent such velocities to be expressed.

This means that it is not the force proper that moves, being in permanent action by structure, irrespective of the distance separating the particles, and that it is the kinetic energy induced by the force that has a limited velocity, less than $\mathbf{c}$ for kinetic energy induced in massive charged particles, and $\mathbf{c}$ for amounts of translational kinetic energy liberated as free moving electromagnetic photons, when accelerating massive charged particles are suddenly stopped in their motion when forced to stabilize in some reversible least action electromagnetic equilibrium state. The part played by the translational kinetic energy component with respect to velocity of the complete particle is analysed in separate references $[2,9]$.

Let us consider a free moving electron that just appeared by production of an electron/positron pair from a single $1.022 \mathrm{MeV}$ electromagnetic photon [7], a free moving electron that will now be attracted for the first time of its existence by a proton to form a hydrogen atom.

The Coulomb equation reveals that at the Bohr radius $\left(r_{0}=5.291772083 \mathrm{E}-11 \mathrm{~m}\right)$, which also corresponds to the most probable stabilization distance from the proton that can be calculated from the statistical spread of the wave function in Quantum Mechanics, an amount of unreleasable kinetic energy exactly equal to $27.2 \mathrm{eV}$ is permanently induced at this distance from the proton:

$$
\mathrm{E}=\frac{1}{4 \pi \varepsilon_{\mathrm{o}}} \frac{\mathrm{e}^{2}}{\mathrm{r}_{0}}=4.359743805 \mathrm{E}-18 \mathrm{~J} \text { (That is, } 27.2 \mathrm{eV} \text { ) }
$$

The same amount of energy can also be calculated by integrating all of the kinetic energy that the electron accumulates while accelerating to reach the proton rest orbital (see equation (10) further on), including the part of this kinetic energy that converts to the momentary relativistic mass increment that can be related to the relativistic velocity that an electron would be freely moving at when induced with this amount of energy [9].

At our macroscopic level, the total amount of kinetic energy that animates a body can be experimentally confirmed only indirectly, by 
calculating the kinetic energy that the body longitudinally releases when it is forced to come to a dead stop, leaving behind only the energy making up the rest mass of the body (its mass when its velocity is zero), as it communicates to the target all of the kinetic energy that it possessed in excess of its rest mass.

This process is also true for the electron as it is captured in electromagnetic equilibrium at the mean rest orbital of a hydrogen atom, except that in such a naturally occurring stable state, the electron is unable to communicate "all" of the kinetic that it possesses in excess of its rest mass.

The classical equation used to calculate the energy in excess of the rest mass of a body or particle for non relativistic velocities is $\mathrm{E}_{\mathrm{k}}=\left(\mathrm{mv}^{2}\right) / 2$ (an equation that leaves out the part that converts to the momentary relativistic mass increment, which is infinitesimal for such low velocities), and more precisely with $E_{k}=m^{2}(\gamma-1)$ when relativistic velocities are reached, which takes into account the added kinetic energy amount required to propel at this velocity the total momentary relativistic mass that it possesses when being stopped.

In both these traditional equations, however, the value of $\mathrm{E}_{\mathrm{k}}$ represents only the amount of "translational" (aka unidirectional) kinetic energy that propels the rest mass of the body in the first case, and its momentary relativistic mass (rest mass plus momentary velocity related relativistic mass increment) in the second case, which is the cumulative effect of a force measured by its integrated effect over time. But in both equations, $\mathrm{E}_{\mathrm{k}}$ does not include the kinetic energy that converts to the momentary relativistic mass increment:

\section{$\int \mathrm{Fdt}$}

This is easily verified by using non relativistic velocity 2187691.253 $\mathrm{m} / \mathrm{s}$ with equation $\mathrm{E}_{\mathrm{k}}=\left(\mathrm{mv}^{2}\right) / 2$ to calculate the translational energy of the electron on the Bohr orbit:

$$
\mathrm{E}_{\mathrm{k}}=\frac{\mathrm{mv}^{2}}{2}=2.179871904 \mathrm{E}-18 \mathrm{~J} \text { (That is, } 13.60569173 \mathrm{eV} \text { ) }
$$

And by using relativistic velocity $2187647.561 \mathrm{~m} / \mathrm{s}$ with relativistic equation $\mathrm{E}_{\mathrm{k}}=\mathrm{mc}^{2}(\gamma-1)$ :

$$
\left.\mathrm{E}_{\mathrm{k}}=\mathrm{mc}^{2}(\gamma-1)=2.179873 \mathrm{E}-18 \mathrm{~J} \text { (That is, } 13.60569857 \mathrm{eV}\right)(7)
$$

which is the translational energy of the electron on the mean ground state orbital of the electron in an isolated hydrogen atom.

It can be observed that both values (non-relativistic and relativistic) are infinitesimally close to each other due to the fact that although measurable, the relativistic velocity of the electron at this energy level is in the low relativistic range, which explains why the non-relativistic values that can be calculated from the Bohr model are precise enough as a basis for all electronic orbitals calculations.

The doubled amount of kinetic energy first mentioned, comprising both the unidirectional translational propelling energy plus the amount that momentarily converts to the corresponding relativistic mass increment, corresponds in reality to the expression $\mathrm{Fr}=\mathrm{E}=\mathrm{mv}^{2}$, which happens to be the amount of energy that can be calculated with the fundamental acceleration equation $\mathrm{F}=\mathrm{ma}$, which is equated to Coulomb equation (4) in undergrad textbooks, as demonstrated in reference [19].

This last quantity is the kinetic energy that an inverse square acting force adiabatically accumulates as measured by the integrated effect of the force over space (that is, distance), since kinetic energy is induced by the force as a function of the inverse square of the distance between the particles, and not as a function of the time involved: $\int \mathrm{Fdx}$

This last quantity, that Leibnitz considered to be the true measure of "the effects of application of a force" [16] which is the definition of "acceleration", amounts to twice the translational kinetic energy $\left(\mathrm{E}_{k}\right.$ quoted previously). So, it is then the amount of kinetic energy that accumulates by integration as a function of distance in agreement with Leibnitz that we will be measuring here. The general formula for this relation is then:

$$
\begin{aligned}
& \int_{r>0}^{\infty} \mathrm{F} \cdot \mathrm{dr} \text {, to be applied to the Coulomb force equation } \\
& \mathrm{F}=\mathrm{k} \frac{\mathrm{e}^{2}}{\mathrm{r}^{2}}
\end{aligned}
$$

Integrating the Coulomb equation for electrostatic force and calculating the total amount of kinetic energy that accumulates during the acceleration sequence, including the energy that progressively converts to the relativistic mass increment, before finally arriving at the Bohr orbit, we obtain:

$$
\mathrm{E}=\int_{\mathrm{r}_{0}}^{\infty} \frac{1}{4 \pi \varepsilon_{0}} \frac{\mathrm{e}^{2}}{\mathrm{r}^{2}} \cdot \mathrm{dr}=0-\frac{1}{4 \pi \varepsilon_{0}} \frac{\mathrm{e}^{2}}{\mathrm{r}_{0}}=-4.359743805 \mathrm{E}-18 \mathrm{~J}
$$

We note here that the amount of 4.359743805 E-18 joules $(27.2 \mathrm{eV})$ obtained corresponds to double the energy liberated as a bremmsstrahlung photon escaping in physical reality as the electron stabilizes on its least action mean rest orbital about the proton. Now why is there this difference? And why is it that the full amount of 27.2 $\mathrm{eV}$ still remains present at the mean ground orbital even after a $13.6 \mathrm{eV}$ photon has been released?

As the electron arrives at the mean rest orbital, it is animated by an amount of translational kinetic energy equal to $13.6 \mathrm{eV}$ vectorially directed towards the proton at whatever angle the local electromagnetic circumstances allowed, plus an equal amount of $13.6 \mathrm{eV} / \mathrm{c}^{2}$ that progressively converted to the corresponding velocity related relativistic mass increment during the acceleration phase.

But since the amount of energy induced by the Coulomb force at this distance from the nucleus can in no way be different from $27.2 \mathrm{eV}$, it can be forcefully concluded that as the $13.6 \mathrm{eV}$ photon is evacuated, carrying away the unidirectional translational energy that the electron previously accumulated during the acceleration phase, a replacement $13.6 \mathrm{eV}$ amount of unidirectional kinetic energy has to synchronously be induced by the permanently acting Coulomb force, an energy whose vectorical direction will now be expressed according to the local electromagnetic equilibrium.

The total mass of the electron will now be stabilized at the following value, made up of the invariant rest mass of the electron plus a carryingenergy related mass increment equal to $13.6 \mathrm{eV} / \mathrm{c}^{2}$ :

$$
\mathrm{m}=\mathrm{m}_{0}+\frac{\mathrm{E}_{\left(\mathrm{r}_{0}\right)}}{2 \mathrm{c}^{2}}
$$

Plus the newly induced replacement amount of unidirectional 13.6 eV kinetic energy.

It can easily be verified that the mass calculated with equation (11) is exactly equal to the relativistic mass of a localized electron deemed to be moving at relativistic velocity $2187647.561 \mathrm{~m} / \mathrm{s}$.

So, we observe that the acceleration sequence generated a total amount of $40.8 \mathrm{eV}$ of kinetic energy; $13.6 \mathrm{eV}$ of which is forcibly ejected upon arrival at the mean rest orbital, due to its longitudinal inertia, metaphorically speaking, like a passenger being ejected from a car 
hitting a wall, with the remaining $27.2 \mathrm{eV}$ statically remaining induced in the electron for as long as it will remain in this equilibrium state, varying only slightly as the electron axially hovers about the mean rest orbital within the allowed statistical spread, as previously described.

It is to be noted that all electromagnetic photons coming from deep space or local earthly processes are known to have been emitted through the very same bremmsstrahlung process, from the lowest energy photons, emitted by electrons moving to closer locations towards atomic nuclei, to the most intense gamma ray photons emitted by fissioning or fusing atomic nuclei or other nuclear rearrangements. Let us note also that all photons emitted during scattering events as elementary particles collide with each other also enter the bremmsstrahlung category, such as when cosmic "rays", mostly made up of hyper-energetic protons coming from deep-space, as previously mentioned, collide with atoms or molecules in the Earth's atmosphere or ground level.

\section{Linking the Natural Adiabatic Heat Increase with Depth in the Earth Mass with Atomic Orbitals Compression}

We can now put in perspective that the unreleasable amount of $27.2 \mathrm{eV}$ of energy which is permanently induced in the electron for as long as it remains stabilized on the natural ground state orbital of a hydrogen atom is in a situation slightly different from the energy induced by adiabatic compression in the Earth mass, which is known to permanently maintain an estimated temperature of about 5100 degrees Kelvin at the center of the Earth [1].

It must be put in perspective here that the hydrogen mean rest orbital corresponding to the Bohr radius $(r=5.291772083 \mathrm{E}-11 \mathrm{~m})$, is the radius of the "uncompressed" hydrogen atom. And the same goes for all standard calculated orbitals in all atoms of the periodic table [20].

It is at this point that the adiabatic increase in heat with increasing depth inside the Earth can be related to atomic orbitals compression. It is well established that heat in liquids and solid bodies is directly related the amount of energy in excess of the unreleasable energy that the Coulomb force induces at the discrete distance at which the local electromagnetic equilibrium forces the electron to reside in atoms.

At normal pressure at the surface of the earth, this excess excitation of electrons on their orbitals is due to energy transfers between atoms by means of convection, conduction or radiation. In the case of increasing compression with increasing depth in the Earth, neither convection, conduction nor radiation can be directly at play. The only possible cause of adiabatic heat increase with increasing pressure of liquids and solids in the Earth mass is related to atoms being compressed into smaller volumes, which shortens the distances at which electrons are stabilized at with respect to nuclei.

Since energy is induced as a function of the inverse square of the distance between electrons and nuclei, shorter distances will cause increased energy to be induced in electrons at these shorter distances.

For example, if a hydrogen atom could hypothetically be found at the very center of the Earthand be induced by compression with the energy corresponding to $5100^{\circ} \mathrm{K}$, then the radius at which the electron would be forced at in such a hydrogen atom can be easily calculated.

Of cource all such calculations are approximate to some degree, even for the best estimation methods described in reference [1], but by using the energy required to increase one mole of Hydrogen $\left(\mathrm{H}_{2}\right)$ by 1 degree Kelvin, a ballpark figure can easily be obtained, even if highly approximate.
Considering that 1 mole of $\mathrm{H}_{2}$ has a mass of 2.0158 gram and that 28.836 joules of energy is required to raise its temperature by $1^{\circ} \mathrm{K}$, we find that an excess amount of energy of $0.7621 \mathrm{eV}$ will be induced at the compressed orbital of an hydrogen atom hypothetically located at the center of the Earth, which will establish the energy of its carrierphoton at $27.2+0.7621 \mathrm{eV}=27.9621 \mathrm{eV}$, which converted to joules gives $4.480021845 \mathrm{E}-18 \mathrm{j}$, to be compared to $4.359743805 \mathrm{E}-18 \mathrm{j}$ at the uncompressed rest orbital.

Isolating the radius in the Coulomb equation (see equation (4)), and using this increased amount of Coulomb force induced energy, we obtain the following shortened radius for the compressed mean hydrogen rest orbital hypothetically located at the center of the Earth:

$$
\mathrm{r}=\frac{1}{4 \pi \varepsilon_{\mathrm{o}}} \frac{\mathrm{e}^{2}}{\mathrm{E}}=5.1497 \mathrm{E}-11 \mathrm{~m}
$$

To be compared with the uncompressed Bohr radius of 5.291772E$11 \mathrm{~m}$.

So we can well imagine how much more compressed atoms will be at the center of Jupiter for example (317.8 times the mass of the Earth), or the Sun (333000 times the mass of the Earth), and how much hotter the center of these celestial bodies have to be.

Even if one could expect that this heat would diffuse over time by conduction right to the surface, it has been demonstrated that no energy induced by adiabatic compression can diffuse by conduction into less compressed areas in an isolated system. Indeed, as the pressure diminishes with diminishing distance from the surface, less energy will simply be induced in electrons as atomic volumes increase in sync and the distance between electrons and nuclei also increases.

In all adiabatic compression experiments carried out at our macroscopic level with isolated systems, the heat generated at maximum compression diminishes as pressure diminishes and will return to initial temperature as initial pressure is restored, demonstrating that the process is totally reversible. Furthermore, diminishing the pressure further causes the temperature in a closed system to fall below ambient temperature. It is this discovery that allowed the development of various types of refrigeration systems, by allowing the cyclically generated compression heat to be diffused in the environment before reducing the pressure in a closed system. Among other useful applications, another example is the use of adiabatically generated compression heat to ignite fuel in diesel engines.

Indeed, even the energy amount of $13.6 \mathrm{eV}$ that must be provided to chase an electron away from the rest orbital of a hydrogen atom seems to also sort of diminish in quantity in the same manner as the distance increases between the electron and the proton, to theoretically reach zero at infinity, an amount that will re-accumulate again as this particular electron accelerates again to be re-captured by a proton.

If we re-examine the example of a $1 \mathrm{~kg}$ body resting on the Earth's surface, this body is in a state of least action energy equilibrium, just like the matter located at the center of the Earth.

Let us now become aware that from a strict electromagnetic perspective, this $1 \mathrm{~kg}$ body, as well as the matter of which the ground is made at the surface of the Earth is ultimately only made up of atoms, whose ultimate building blocks are electrons, up quarks and down quarks, which are the only scatterable point-like behaving charged and massive particles that were ever detected in atomic structures. All of them being charged, it becomes obvious at this point that all of these point-like behaving particles are permanently subject to the Coulomb 
force, and that his force will be in action between the $1 \mathrm{~kg}$ mass and the Earth, finding themselves in the same situation as an electron being attracted by a proton by the Coulomb force.

If we force this body to move away from the surface towards an equilibrium state of lesser energy, a height of 1 meter as in our example, by providing it with the 9.78 joules of energy that its component particles initially accumulated and released as they reached the surface for the first time in the past from this 1 meter height, all considerations lead to conclude that this 9.78 joules will progressively and adiabatically "diminish" as the body is moved up towards this 1 meter height, resolving to this energy being "expended" as "work" being done, to reach this height.

When the body becomes momentarily immobile at this 1 meter height, it seems difficult not to conclude that this energy will now have been completely "expended". More precisely, that the body will be left with exactly the unreleasable kinetic energy induced by the Coulomb force in action between the point-like behaving ultimate components of the $1 \mathrm{~kg}$ mass and those of the Earth at this higher distance from the Earth and that would be remain stable at this value for as long as the body is made to remain at rest at this height.

As previously put in perspective, with respect to the principle of energy conservation, when the $1 \mathrm{~kg}$ body re-accelerates towards the Earth, all measurements confirm that it will re-accumulate exactly 9.78 joules of translational energy, that will be released to the environment as the body reaches the ground, now again statically exerting a pressure of $1 \mathrm{~kg}$ against the surface, in a process exactly similar to that of the electron releasing $13.6 \mathrm{eV}$ as it is stopped in electromagnetic equilibrium on the ground state orbital.

So, transposing the adiabatic process just analyzed in the case of the $9.10938188 \mathrm{E}-31 \mathrm{~kg}$ mass of the electron re-accumulating energy as it reaccelerates towards the ground orbital equilibrium state in a hydrogen atom, it can be asserted that during the re-acceleration phase of the $1 \mathrm{~kg}$ mass, it will in reality accumulate a total of 29.34 joules of energy, that is, 9.78 joules of which it will release, and the remaining 19.56 being adiabatically unreleasable, and made up of 9.78 joules of unidirectional energy, translationally directed towards the center of the Earth and corresponding to the pressure that the $1 \mathrm{~kg}$ mass applies against the ground, and the remaining 9.78 joules converted to a mass increment of $9.78 / \mathrm{c}^{2}=1.088171755 \mathrm{E}-16 \mathrm{~kg}$, which is an amount 13 orders of magnitude smaller than 1 gram, as mentioned at the beginning of this analysis.

The manner in which such an adiabatically unreleasable amount of 19.56 joules would diminish as the $1 \mathrm{~kg}$ body is raised to a height of 1 meter, and then be re-accumulated as the mass returns to its initial least action rest state on the ground, in a manner identical to the adiabatically unreleasable $27.2 \mathrm{eV}$ would diminish as the electron escapes from the proton and is re-accumulated again as it re-accelerates to be recaptured, is what supports the idea that such systems are involved in reversible adiabatic processes.

\section{Potential Uses of Initial Irreversibly Induced Energy}

All of these considerations come in support of the idea that a newly created particle that never was "forced away" from some more intense energy equilibrium state, will adiabatically and irreversibly accumulate for the first time of its existence, the energy required to reach that more energetic equilibrium state from a less energetic equilibrium state. Now, what possible use could such newly created energy be put to, if these conclusions were confirmed? Not much use indeed in the case just examined, since $13.6 \mathrm{eV}$ amounts to barely $2.1798719 \mathrm{E}-18$ joules, which means that to amount to 1 joule, we would then need to put to work one hundred million billion newly created electrons! Setting up useful control of such astronomical numbers of occurrences seems utterly impossible to even consider, given that 9.78 joules must be expended to raise a mass of $1 \mathrm{~kg}$ to a height of one meter above ground!

The question that now comes to mind is the following: Could there exist some other natural least action electromagnetic equilibrium state or states that electrons could reach that would be more energetic than the most energetic least action equilibrium state involving electronic orbitals? More generally formulated: What is the most energetic least action equilibrium state that an electron can irreversibly adiabatically accelerate to in nature?

It is to be noted that in the crowd of particles that populate the Standard Model, only 4 massive and stable particles exist in the whole collection, which are the electron, the positron, the proton and the neutron, all other particles being fleetingly existing metastable particles that swiftly release excess energy during well documented decay processes, and that ultimately always leave behind one or other of this very restricted set of 4 really stable massive particles, besides nonmassive electromagnetic photons and neutrinos.

We have ample experimental proof that electrons and positrons can be created from the destabilization of photons of energy $1.022 \mathrm{MeV}$ or more by a process involving a possible mechanics such as analyzed and explained in [7]. But no clear-cut mechanical process was ever established, nor experimentally proven that could logically explain how protons and neutrons come into being.

The only experimental telltale that we really have as to their possible origin, comes from the extensive production of hadrons (mesons and baryons, including protons and neutrons) in numerous experiments carried out with colliding beams of electrons and positrons at the colliding-beam SPEAR facility of the Stanford Linear Accelerator in the 1970's [21].

This suggested the possibility that the up and down quarks first detected in the 1966-1968 period could possibly be electrons and positrons that would somehow mutually capture in threesomes to then accelerate until they end up stabilizing as the inner point-like behaving components of protons and neutrons, despite the differences in verified masses and sign intensities of the charges of electron and down quark on one hand, and those of positron and up quark on the other, that would remain to be explained.

Another telltale is the facts that up and down quarks always behave point-like, just like positrons and electrons.

Of course, the immediate objection to the possible existence of such a process was that the principle of conservation of energy absolutely precludes the possibility that three particles with masses $0.511 \mathrm{MeV} /$ $\mathrm{c}^{2}$, for a total initial mass of $1.533 \mathrm{MeV} / \mathrm{c}^{2}$ could transform into a stable mass of $938 \mathrm{MeV} / \mathrm{c}^{2}$ such as that of the proton or the neutron, without burrowing energy from the surrounding environment, a burrowing that experimental data clearly shows did not happen during these experiments. But we just saw that the principle of conservation was never defined to completely integrate adiabatic processes, and leaves out by definition the case of newly created massive and charged particles.

However, all attempts at developing a logical mechanics of conversion that would fit within the confines of the traditional 4-dimensional space time geometry that underlies all currently accepted theories were fruitless. Only when the more elaborate 3 -spaces 
space geometry described in reference [2] was conceived of, did such a possible mechanics of conversion begin to make sense while remaining Maxwell equations compliant.

This mechanics coherently covers all processes from electromagnetic photon generation, as described in reference [2], to electron-positron pair production from electromagnetic photons of energy $1.022 \mathrm{MeV}$ or more as described in reference [7], to the production of protons and neutrons with electrons and positrons as point-like behaving building blocks, that would mutually captured in e+ e+ e- and e- e- e+ triads, with insufficient energy to escape mutual interaction as described in reference [8].

The proposed mechanics also allows reconciling the sign intensity differences of the charges and the mass differences between electron and down quark, and between positron and up quark, in full agreement with already well understood processes [8].

The reason why up and down quarks would settle at these wellknown mean distances of the order of $1.2 \mathrm{E}-15 \mathrm{~m}$ from the center of the triads can of course only be due to the same electromagnetic equilibrium mechanics previously clarified for the hydrogen atom, involving the interplay of both the inverse square electric interaction law and the magnetic inverse cube interaction law. This mechanics is explained in reference [3].

The newly created energy liberated by each such nucleon genesis occurrence amounts to three bremmsstrahlung photons of $155 \mathrm{MeV}$ each, for a total of $465 \mathrm{MeV}$, plus a stable relativistic unreleasable mass increase of $938-1.533=936.467 \mathrm{MeV} / \mathrm{c}^{2}$, for a grand total energy gain of $1401.467 \mathrm{MeV}$, that is, $1,401,467,000 \mathrm{eV}$ or $2.245 \mathrm{E}-10$ Joules, which is 34.35 million times more energy than the $40.8 \mathrm{eV}$ gained from the initial irreversible adiabatic acceleration of a newly created electron to the least action orbital of a hydrogen atom.

In the case of nucleons, contrary to the unreleasable $27.2 \mathrm{eV}$ permanently induced at the hydrogen ground state radius, but that will diminish if the electron is moved away from the rest orbital, the unreleasable $936.467 \mathrm{MeV} / \mathrm{c}^{2}$ energy/mass is permanently stabilized in this new electromagnetic equilibrium state. This is why this newly created energy/mass remains available as new permanently usable energy in the form of mass. This reduces the number of nucleon genesis occurrences required to amount to 1 Joule, to 4.454 billion.

This may seem like much, but such figures are well within the usual amounts of particles that are beamed in high energy accelerators in quite reasonable time frames. For example, the collision rate at the LHC accelerator easily reaches about 2.4 billion per minute.

\section{Production of Protons and Neutrons in Nature}

Now if nucleons have been, and possibly still are, produced in nature by such first time irreversible adiabatic acceleration processes involving electrons and positrons, shouldn't we have observed this phenomenon already?

The answer is of course "yes". But since the possibility never was seriously considered before, it may well have been observed without having been recognized for what it really was. For example, the stable 200 fold increase in energy in the Sun's corona with respect to the photosphere, despite huge and constant energy losses due to CMEs and exchanges with the photosphere, has intrigued researchers ever since the corona was discovered [23]. It so happens that the ambient energy level that would be maintained in such a plasma, if the process being considered here was somehow continuously established as some form of low level non-explosive chain reaction, would stabilize precisely in the proper range [17]. Also, the proton-proton fusion process hypothesized by Gamow that has been assumed for the past 70 years as fueling fusion in stars never could be experimentally reproduced due to the insurmountable electrostatic repulsion between protons, repulsion hypothetically deemed surmountable in the center of stars only by pressure and temperature being applied. This process never could be proven in any of the attempted high pressure hydrogen fusion experiments. Indeed, all successful fusion experiments show that neutrons absolutely need to be involved in the process.

In fact, the only successful cases of sustained chain reaction hydrogen fusion on the Earth involved massive amounts of thermal neutrons momentarily produced by the fission detonators (atomic bombs), during experimental testing of unfortunately military motivated hydrogen bomb explosions. The nucleon generation process envisioned here, on its part, stochastically results in the theoretical production of as many thermal neutrons as protons, which would de facto allow easily reproducible proton-neutron-proton fusion.

It becomes then entirely possible to consider that the main source of energy in star masses could be a nucleogenesis process such as the one envisioned here, and that it could even be the real triggering initial ignition mechanism in primordial hydrogen masses reaching sufficient central adiabatic pressure [18].

What leads to this conclusion is that adiabatic compression suffered by atoms' electronic escorts as pressure increases with depth in large celestial bodies such as stars masses, is sufficient in their central areas for the unreleasable energy induced in the electrons of hydrogen atoms for their carrier-photon to reach the $1.022 \mathrm{MeV}$ threshold level at a distance of less than $0.2 \mathrm{E}-15$ from the proton.

Now, the energy in excess of the rest mass of a moving elementary particle such as an electron, has clearly been established as having the very same electromagnetic characteristics as those of a free moving photon [2,9], the only difference being that in the case of a moving electron, this excess energy has to "carry", so to speak, the massive electron that it is associated with, hence its name of carrier-photon. It can then be fully expected that this carrier-photon, if it were to reach the $1.022 \mathrm{MeV}$ threshold, would also be susceptible to decoupling [18]

Protons having been experimentally confirmed to have a radius of about $1.2 \mathrm{E}-15 \mathrm{~m}$, this means that the $1.022 \mathrm{MeV}$ carrier-photons of the related electrons, now located at about $0.2 \mathrm{E}-15 \mathrm{~m}$ from the proton, find themselves in the same grazing circumstance with respect to their massive proton companions as do free moving $1.022 \mathrm{MeV}$ electromagnetic photons that would be grazing massive particles.

The complete sequence of proto-stars ignition from decoupling electron carrier-photons revealed by the 3 -spaces model is analyzed in a separate paper [18]

\section{Control and Use of Initial Irreversible Adiabatic Accel- eration Processes}

It is not difficult to imagine what would become possible if we were able to consistently manufacture protons and neutrons from electronpositron pairs generated from the decoupling of pure energy massless $1.022 \mathrm{MeV}$ electromagnetic photons, which amounts to manufacturing matter from energy with processes that are many orders of magnitude more efficient than any of the reversible processes currently in use to harness usable energy.

These processes could finally provide us with an easy way to 
control hydrogen fusion with continuous flows of thermal neutrons that could be produced in time-frames compatible with such control, at long last making available the huge amounts of energy that hydrogen fusion promises. Only experimentation will show whether such a nucleogenesis process can free sufficient energy to become itself a primary source of usable energy.

The most mind boggling perspective however, is the potential development of a starship engine ejecting mass newly created from massless electromagnetic energy that could provide sufficient energy for continuous trust, possibly up to the $1 \mathrm{~g}$ range, which would do away with the health issues inherent to inertial weightless space travel, without the need to carry large quantities of propellant, which would bring the farthest reaches of the solar system within immediate reach, and travel to the nearest stars largely within the span of a human life [18], because if nucleons rest masses are demonstrated as varying with local gravitational intensity by the easy to carry out low-tech experiment proposed further on, which would be a dead giveaway that variable relativistic velocities or alternately variable relativistic energy levels are involved inside nucleons, then the Special Relativity Theory theoretical space-time dilation will not be an issue anymore [18].

Two different possible nucleogenesis methods are within our current technical abilities to use. The first involves driving a beam of electromagnetic photons having the least amount of energy possible over the $1.022 \mathrm{MeV}$ threshold against target material to be identified, possibly an intersecting second $1.022 \mathrm{MeV}$ photon beam, or a proton beam, which would produce quantities of thermal electron-positron pairs having insufficient energy to escape each other's close interaction, which should allow triads to be formed and start the irreversible initial threesome acceleration sequences.

The second method involves accelerating electrons (or positrons) to a minimum velocity of $259627884 \mathrm{~m} / \mathrm{s}$, which is $86.6 \%$ of the speed of light, which is an easily reached velocity in high energy accelerators and/or possibly the new FEL wigglers that are entering service on many high energy accelerator sites, and having them meet target materials to be identified, possibly a proton beam which would cause their 1.021 $998 \mathrm{MeV}$ carrier-photon to destabilize with no residual energy left to escape the moving carried particle and would immediately convert to a pair of electron-positron that would form an accelerating triad with the now immobilized carried particle. Beams of electrons would produce neutrons and beams of positrons would produce protons.

\section{Experimental Confirmation}

Finally, is there some easy means to experimentally confirm whether or not protons and neutrons can come into being in this manner?

\section{The answer is yes.}

If protons and neutrons are in reality triads of electrons and positrons that accelerated until they reached a stable least action electromagnetic equilibrium within a volume whose radius is in the $1.2 \mathrm{E}-15 \mathrm{~m}$ range, this would mean that the better part of nucleon masses can only be relativistic in nature since the verified possible mass ranges of the up and down quarks making up their scatterable inner structure have been experimentally found to amount to about $2 \%$ for the proton and $2.4 \%$ for the neutron as shown in Table $1[8,20]$.

This relativistic mass must then be related to the highly relativistic velocities and/or carrying-energy levels that the up quarks (accelerated positrons) and down quarks (accelerated electrons) have to maintain on the very short gyroradii closed orbits on which this electromagnetic equilibrium state forces them to stabilize. These velocities and all other related parameters are explained in reference [8].

Given that relativistic velocities or energy levels would be involved, the measurable masses of protons and neutrons would be directly dependent on the local Coulomb field gradient. This would mean that the distances separating the 3 charged quarks within each nucleons in the nuclei of atoms located at the center of large accumulations of matter will increase, due to the charged quarks being more strongly pulled outwards by Coulomb attraction at shorter range from large quantities of other charged up and down quarks belonging to nucleons of nuclei of other atoms that populate the immediately surroundings.

Then if small quantities of atoms are taken away from a large mass, these distances between the charged quarks within their nucleons are bound to shorten somewhat due the diminishing outward pull of the charged quarks of other atoms now being located further away as a function of the inverse of the square of the distance, which unavoidably will cause their relativistic velocities and/or carrying-energy levels to increase within nucleons, which in turn will cause an increase of the relativistic component of their mass.

This also means that less densely packed nuclei such as those of lithium or magnesium for example would have a nucleon contraction gradient towards maximum density as local Coulomb field gradient intensity decreases that would be more pronounced than that of denser elements, such as uranium or osmium, given that they contain much fewer nucleons in volumes of about the same order. The diameter of denser atoms, including electronic escorts being estimated to be only about 3 times that of hydrogen, the ratio between the lowest density and highest density metals will be lower yet, meaning that the nucleons of less dense elements should contract more rapidly than those of denser elements towards their limit as they are lifted in altitude away from the surface of the Earth.

This can be verified in a very simple manner, that was proposed already in an article submitted to the "Bureau International des Poids et Mesures" (BIPM) and to the "National Institute of Standards and Technology" (NIST) at the beginning of March of 2011. Only an equal-arm balance would be required to conduct the experiment, in which two equal masses of elements of widely different densities would be set in perfect equilibrium at ground level, or better yet, at the bottom of the deepest mine shaft possible. This assembly would then be lifted in altitude.

Why not $10 \mathrm{~km}$, as was done with the first cesium atomic clocks experiment? If nucleons contraction gradients really are different for low and high density elements, as hypothesized here, then the side holding the low density element should go down as altitude increases, showing that the less dense element is now becoming more massive than the higher density element.

This would prove out of any possible doubt that varying relativistic velocities and/or carrying-energy levels are involved inside nucleons, which would definitely give substance to the possibility that nucleons could come into being by means of an irreversible initial adiabatic acceleration process such as the one proposed here.

Other than this easily carried out low-tech experiment, a high-tech experiment could also be conducted that would directly confirm that up and down quarks actually are electrons and positrons whose masses and charge characteristics are warped into these altered states by the stresses imposed by these most energetic least action equilibrium states that electrons and positrons can reach in nature.

Since the making or neutrons and protons from triads of electrons 
and positrons would by definition be reversible adiabatic processes just like the making of a hydrogen atom by the stabilization of an electron on the least action rest orbital about a proton, and that it is possible to liberate this electron by providing it with the same amount of $13.6 \mathrm{eV}$ energy that it liberated while stabilizing, it should similarly theoretically be possible to free the electron and 2 positrons captive in an immobilized proton, by causing it to simultaneously absorb 3 photons of energy slightly higher than $154.8696007 \mathrm{MeV}$, which is the translational energy that each quark had to liberate as the proton came into being.

Similarly for the neutron, the theoretical simultaneous absorption of 3 photons of energy slightly higher than $155.2289185 \mathrm{MeV}$ should free the captive positron and 2 electrons, causing the non-releasable adiabatic energy that they accumulated as they stabilized in the neutron configuration to adiabatically reduce to zero in this case also.

In practice however, considering the difficulty inherent in producing and precision guiding such high energy photons, it is possible to consider using more numerous lesser energy photons amounting to or exceeding the required $465 \mathrm{MeV}$ liberation energy being simultaneously absorbed by an immobilized target proton, coming from arrays of high power lasers.

The success of such an experimental freeing of the inner scatterable components of a proton as free moving electrons and positrons, accompanied by the disappearance of the adiabatically induced unreleasable energy involved, would constitute the physical proof out of any doubt of the reality of the processes described in the present analysis. That is, by experimentally proving that up and down quarks really are only positrons and electrons whose masses and charge characteristics are warped into these altered states by the stresses imposed by these most energetic least action equilibrium states that electrons and positrons can reach in nature.

\section{Conclusion}

Considering the relative simplicity of implementation of the experiments that could confirm whether or not the first initial irreversible acceleration sequence of newly created elementary particles is subject to the principle of conservation, and the potentially unlimited energy source that could become available from controlling the process, it is to be hoped that the physics community will become interested sooner than later to having these experiments carried out.

\section{References}

1. Lowrie W (2007) Fundamentals of Geophysics, Second Edition, Cambridge University Press.

2. http://www.omicsonline.com/open-access/on-de-broglies-doubleparticlephoton-hypothesis-2090-0902-1000153.php?aid=70373

3. http://www.omicsonline.com/open-access/on-de-broglies-doubleparticlephoton-hypothesis-2090-0902-1000153.php?aid=70373. International Journal of Engineering Research and Development 7: 50-66.

Citation: Michaud A (2016) On Adiabatic Processes at the Elementary Particle Level. J Phys Math 7: 177. doi:10.4172/2090-0902.1000177
4. Feynman R (1949) Space-Time Approach to Quantum Electrodynamics. Phys Rev 76: 769-775.

5. Breidenbach M (1969) Observed Behavior of Highly Inelastic Electron-Proton Scattering. Phys Rev Let 23: 935-939.

6. Kotler S, Akerman N, Navon N, Glickman Y, Ozeri R (2014) Measurement of the magnetic interaction between two bound electrons of two separate ions. Nature magazine 510: 376-380.

7. Michaud A (2013) The Mechanics of Electron-Positron Pair Creation in the 3-Spaces Model. International Journal of Engineering Research and Development 6: 36-49.

8. Michaud A (2013) The Mechanics of Neutron and Proton Creation in the 3-Spaces Model. International Journal of Engineering Research and Development 7: 29-53.

9. Michaud A (2013) From Classical to Relativistic Mechanics via Maxwell. International Journal of Engineering Research and Development 6: 01-10.

10. Marmet $P$ (2003) Fundamental Nature of Relativistic Mass and Magnetic Fields. International IFNA-ANS Journal, Kazan State University.

11. Kaufmann W (1903) Über die "Elektromagnetische Masse" der Elektronen, Kgl. Gesellschaft der Wissenschaften Nachrichten. Mathem-Phys Klasse 91-103.

12. Michaud A (2013) On the Electron Magnetic Moment Anomaly. International Journal of Engineering Research and Development 7: 21-25.

13. Stodolna AS, Rouzée A (2013) Hydrogen Atoms under Magnification: Direct Observation of the Nodal Structure of Stark States. Physical Review Letters.

14. Blackett PMS, Occhialini G (1933) Some photographs of the tracks of penetrating radiation. Proceedings of the Royal Society 139: 699-724.

15. McDonald K (1997) Positron http://journals.aps.org/prl/abstract/10.1103/ PhysRevLett.79.1626

16. Resnick R (1976) Physics. John Wyley \& Sons, New York.

17. Michaud A (2013) The Corona Effect. International Journal of Engineering Research and Development 7: 01-09.

18. Michaud A (2013) Inside planets and stars masses. International Journal of Engineering Research and Development 8: 10-33.

19. Resnick R, Halliday D (1967) Physics. John Wyley \& Sons, New York.

20. Lide DR (2003) CRC Handbook of Chemistry and Physics. (84thedn) CRC Press, New York.

21. Hanson G, Agrams GS (1975) Evidence for Jet Structure in Hadron Production by e+ e- Annihilation. Phys Rev Let 35: 1609-1612.

22. Michaud A (2013) The Expanded Maxwellian Space Geometry and the Photon Fundamental LC Equation. International Journal of Engineering Research and Development 6: 31-45.

23. Aschwanden M (2006) Physics of the Solar Corona, Springer

24. National institute of Standards and Technology, (NIST).

OMICS International: Publication Benefits \& Features

Unique features:

- Increased global visibility of articles through worldwide distribution and indexing

Showcasing recent research output in a timely and updated manner

Special issues on the current trends of scientific research

Special features:

700+ Open Access Journals

$50,000+$ editorial team

Rapid review process

Quality and quick editorial, review and publication processing

Indexing at major indexing services

Sharing Option: Social Networking Enabled

Authors, Reviewers and Editors rewarded with online Scientific Credits

Better discount for your subsequent articles

Submit your manuscript at: http://omicsonline.com/open-access/physical-mathematics.php 